\title{
On a decomposition for pairs of commuting contractions
}

\author{
by \\ ZBIGNiEw BuRDAK (Kraków)
}

\begin{abstract}
A new decomposition of a pair of commuting, but not necessarily doubly commuting contractions is proposed. In the case of power partial isometries a more detailed decomposition is given.
\end{abstract}

1. Introduction. Let $H$ be a complex Hilbert space. Let $H_{0}$ be a subspace of $H$. Then $P_{H_{0}}$ is the orthogonal projection on $H_{0}$. Denote by $L(H)$ the algebra of all bounded linear operators on $H$. An operator $T$ is called completely nonunitary if there is no nontrivial subspace reducing $T$ to a unitary operator. The following decomposition of a contraction with respect to unitarity was given in $[7,11]$.

THEOREM 1.1. Let $T \in L(H)$ be a contraction. There is a unique decomposition

$$
H=H_{u} \oplus H_{\neg u},
$$

where $H_{u}, H_{\neg u}$ are maximal subspaces reducing $T$ such that:

- $\left.T\right|_{H_{u}}$ is a unitary operator,

- $\left.T\right|_{H_{\neg u}}$ is a completely nonunitary operator.

The completely nonunitary part of an operator can be characterized more precisely for certain classes of operators. A well known example is the decomposition of an isometry (Wold [12]), where the completely nonunitary part turns out to be a unilateral shift (of any multiplicity). A larger class of operators with a well characterized completely nonunitary part are power partial isometries. Recall that an operator $T \in L(H)$ is called a partial isometry if $\left.T\right|_{(\operatorname{ker} T)^{\perp}}$ is an isometry. We call $T$ a power partial isometry when every power $T^{n}$ for $n \geq 1$ is a partial isometry. Recall also that a truncated shift of index $k$ is an operator $T$ on a Hilbert space $H \oplus \cdots \oplus H$ ( $k$ times) given by $T\left(x_{1}, \ldots, x_{k}\right)=\left(0, x_{1}, \ldots, x_{k-1}\right)$ for $k \in \mathbb{Z}_{+}$. Halmos and Wallen [5] found the decomposition of a power partial isometry.

2000 Mathematics Subject Classification: Primary 47A05; Secondary 47A15.

Key words and phrases: decomposition, power partial isometries. 
TheOREM 1.2. Let $T \in L(H)$ be a power partial isometry. There is a unique decomposition

$$
H=H_{u} \oplus H_{s} \oplus H_{b} \oplus \bigoplus_{k \geq 1} H_{k},
$$

where $H_{u}, H_{s}, H_{b}$ and $H_{k}, k \geq 1$, are subspaces reducing the operator $T$ such that:

- $\left.T\right|_{H_{u}}$ is a unitary operator,

- $\left.T\right|_{H_{s}}$ is a unilateral shift,

- $\left.T\right|_{H_{b}}$ is a backward shift,

- $\left.T\right|_{H_{k}}$ is a truncated shift of index $k$.

The decompositions given in Theorems 1.1 and 1.2 have a natural generalizations for a pair of doubly commuting operators (i.e., not only do the operators commute, but also each of them commutes with the adjoint of the other). The case of unitary decomposition is given in [9, Proposition 1]:

TheOREM 1.3. Let $T_{1}, T_{2} \in L(H)$ be doubly commuting contractions. There is a unique decomposition

$$
H=H_{u u} \oplus H_{u \neg u} \oplus H_{\neg u u} \oplus H_{\neg u \neg u},
$$

where $H_{u u}, H_{u \neg u}, H_{\neg u u}, H_{\neg u \neg u}$ are subspaces reducing $T_{1}, T_{2}$ such that:

- $\left.T_{1}\right|_{H_{u u}},\left.T_{2}\right|_{H_{u u}}$ are unitary,

- $\left.T_{1}\right|_{H_{u \neg u}}$ is unitary, $\left.T_{2}\right|_{H_{u \neg u}}$ is completely nonunitary,

- $\left.T_{1}\right|_{H_{\neg u u}}$ is completely nonunitary operator, $\left.T_{2}\right|_{H_{\neg u u}}$ is unitary,

- $\left.T_{1}\right|_{H_{\neg u \neg u}},\left.T_{2}\right|_{H_{\neg u \neg u}}$ are completely nonunitary.

The case of doubly commuting power partial isometries was described in [3].

TheOREM 1.4. Let $T_{1}, T_{2} \in L(H)$ be doubly commuting power partial isometries. There is a unique decomposition

$$
H=\bigoplus_{\alpha, \beta \in\{u, s, b\} \cup \mathbb{Z}_{+}} H_{\alpha \beta}
$$

where the $H_{\alpha \beta}$ are maximal subspaces reducing $T_{1}, T_{2}$ such that $\left.T_{1}\right|_{H_{\alpha \beta}}$ belongs to class $\alpha$ and $\left.T_{2}\right|_{H_{\alpha \beta}}$ to class $\beta$ for $\alpha, \beta \in\{u, s, b\} \cup \mathbb{Z}_{+}$. The classes are: class $u$-unitary operators, class $s$-unilateral shifts, class $b$-backward shifts, class $k$-truncated shifts of index $k$ for $k \in \mathbb{Z}_{+}$.

A more universal result generalizing a decomposition of a single operator with respect to any property which is inherited by restrictions to reducing subspaces can be found in [2]. 
2. Decomposition of a pair of contractions. The decompositions given in Theorems 1.3 and 1.4 are entire, but under a strong double commutativity assumption. A natural example of a commuting, but not doubly commuting pair of isometries is $T, T^{2}$, where $T \in L(H)$ is a unilateral shift. For more examples see [1].

For a pair of commuting contractions one can find maximal subspaces $H_{u u}, H_{u \neg u}, H_{\neg u u}$ where the operators are as in Theorem 1.3. However, the orthogonal complement of $H_{u u} \oplus H_{u \neg u} \oplus H_{\neg u u}$ need not reduce the contractions to completely nonunitary operators. Following the definition of a completely nonunitary semigroup of isometries in [10], we say that commuting contractions are a completely nonunitary pair if no proper subspace reduces both operators to unitary operators. Any two operators restricted to $H \ominus\left(H_{u u} \oplus H_{u \neg u} \oplus H_{\neg u u}\right)$ are a completely nonunitary pair. However, the restrictions to $H_{u \neg u}$ or $H_{\neg u u}$ are also completely nonunitary pairs. Therefore we need a more precise definition. We call commuting contractions $T_{1}, T_{2}$ a strongly completely nonunitary pair if there is no proper subspace reducing $T_{1}, T_{2}$ and at least one of them to a unitary operator. It turns out that $H \ominus\left(H_{u u} \oplus H_{u \neg u} \oplus H_{\neg u u}\right)$ is a maximal subspace reducing the contractions $T_{1}, T_{2}$ to a strongly completely nonunitary pair. Precisely, the following decomposition holds.

TheOREM 2.1. Let $T_{1}, T_{2} \in L(H)$ be commuting contractions. There is a unique decomposition

$$
H=H_{u u} \oplus H_{u \neg u} \oplus H_{\neg u u} \oplus H_{\neg(u u)},
$$

where the subspaces $H_{u u}, H_{u \neg u}, H_{\neg u u}, H_{\neg(u u)}$ are maximal with respect to the following:

- $\left.T_{1}\right|_{H_{u u}},\left.T_{2}\right|_{H_{u u}}$ are unitary,

- $\left.T_{1}\right|_{H_{u \neg u}}$ is unitary, $\left.T_{2}\right|_{H_{u \neg u}}$ is completely nonunitary,

- $\left.T_{1}\right|_{H_{\neg u u}}$ is completely nonunitary, $\left.T_{2}\right|_{H_{\neg u \text { u }}}$ is unitary,

- $\left.T_{1}\right|_{H_{\neg(u u)}},\left.T_{2}\right|_{H_{\neg(\text { uu })}}$ is a strongly completely nonunitary pair of contractions.

Proof. Let $\mathcal{H}:=\left\{H_{0} \subset \operatorname{ker}\left(T_{1} T_{2}^{*}-T_{2}^{*} T_{1}\right): P_{H_{0}} T_{i}=T_{i} P_{H_{0}}\right.$ for $\left.i=1,2\right\}$. Recall that a subspace $H_{0}$ reduces an operator if and only if the orthogonal projection $P_{H_{0}}$ commutes with the operator. Thus $H^{d c}=\overline{\operatorname{Span}}\left\{H_{0} \in \mathcal{H}\right\}$ is a maximal subspace reducing the contractions $T_{1}, T_{2}$ to a doubly commuting pair. By Theorem 1.3 applied to $\left.T_{1}\right|_{H^{d c}},\left.T_{2}\right|_{H^{d c}}$, we obtain the decomposition $H^{d c}=H_{u u}^{d c} \oplus H_{u \neg u}^{d c} \oplus H_{\neg u u}^{d c} \oplus H_{\neg u \neg u}^{d c}$. On the other hand, by a simple calculation, or by the Fuglede-Putnam theorem, if one of commuting operators is unitary, they doubly commute. Therefore, $H_{u u}^{d c}, H_{u \neg u}^{d c}$ and $H_{\neg u u}^{d c}$ are maximal subspaces of the entire $H$ reducing $T_{1}, T_{2}$ to operators of the respective types. Thus $H_{u u}=H_{u u}^{d c}, H_{u \neg u}=H_{u \neg u}^{d c}, H_{\neg u u}=H_{\neg u u}^{d c}$. Set 
$H_{\neg(u u)}=H \ominus\left(H_{u u} \oplus H_{u \neg u} \oplus H_{\neg u u}\right)$. By the maximality of $H_{u u}, H_{u \neg u}, H_{\neg u u}$ the restrictions $\left.T_{1}\right|_{H_{\neg(u u)}},\left.T_{2}\right|_{H_{\neg(u u)}}$ are a strongly completely nonunitary pair of contractions.

To show maximality of $H_{\neg(u u)}$, assume that a subspace $H_{0}$ reduces the contractions to a strongly completely nonunitary pair. We may decompose $H \ominus H_{0}=H_{u u}^{\prime} \oplus H_{\neg u u}^{\prime} \oplus H_{u \neg u}^{\prime} \oplus H_{\neg(u u)}^{\prime}$, where $\left.T_{1}\right|_{H_{u u}^{\prime} \oplus H_{u \neg u}^{\prime}}$, $\left.T_{2}\right|_{H_{u u}^{\prime} \oplus H_{\neg \text { 'uu }}^{\prime}}$ are unitary, $\left.T_{1}\right|_{H_{\neg u u}^{\prime}},\left.T_{2}\right|_{H_{u \neg u}^{\prime}}$ are completely nonunitary and $\left.T_{1}\right|_{H_{\neg(u u)}^{\prime}},\left.T_{2}\right|_{H_{\neg(u u)}^{\prime}}$ is a strongly completely nonunitary pair of contractions. The subspace $H_{0} \oplus H_{\neg(u u)}^{\prime}$ also reduces $T_{1}, T_{2}$ to a strongly completely nonunitary pair of contractions. Thus we can assume for convenience that $H \ominus H_{0}=H_{u u}^{\prime} \oplus H_{\neg u u}^{\prime} \oplus H_{u \neg u}^{\prime}$. The maximality of $H_{u u}, H_{\neg u u}, H_{u \neg u}$ implies $H_{u u}^{\prime} \subset H_{u u}, H_{\neg u u}^{\prime} \subset H_{\neg u u}, H_{u \neg u}^{\prime} \subset H_{u \neg u}$. Consequently,

$$
\begin{aligned}
\left(H_{u u} \ominus H_{u u}^{\prime}\right) \oplus\left(H_{u \neg u} \ominus H_{u \neg u}^{\prime}\right) \oplus & \left(H_{\neg u u} \ominus H_{\neg u u}^{\prime}\right) \\
& \subset H \ominus\left(H_{u u}^{\prime} \oplus H_{\neg u u}^{\prime} \oplus H_{u \neg u}^{\prime}\right)=H_{0} .
\end{aligned}
$$

By the definition of a strongly completely nonunitary pair, we deduce that $H_{u u} \ominus H_{u u}^{\prime}=H_{u \neg u} \ominus H_{u \neg u}^{\prime}=H_{\neg u u} \ominus H_{\neg u u}^{\prime}=\{0\}$. The decomposition is unique.

Recall from [8] that a pair of commuting isometries $V_{1}, V_{2}$ is called a weak bi-shift when $\left.V_{1}\right|_{\bigcap_{i \geq 0} \operatorname{ker} V_{2}^{*} V_{1}^{i}},\left.V_{2}\right|_{\bigcap_{i \geq 0}} \operatorname{ker} V_{1}^{*} V_{2}^{i}$ and $V_{1} V_{2}$ are shifts. Theorem 2.1 is a generalization of a result for pairs of isometries given in [8], in particular, a strongly completely nonunitary pair of commuting isometries is a weak bi-shift.

3. Power partial isometries-preliminaries. The following property of partial isometries can be found in [4].

Theorem 3.1. Let $T \in L(H)$. The following conditions are equivalent:

(1) $T$ is a partial isometry,

(2) $T^{*}$ is a partial isometry,

(4) $T T^{*}=P_{\left(\operatorname{ker} T^{*}\right)^{\perp}}$

(3) $T^{*} T=P_{(\operatorname{ker} T)^{\perp}}$,

(5) $T^{*} T T^{*}=T^{*}$

(6) $T T^{*} T=T$.

Note also a trivial but useful property.

Lemma 3.2. Let $T \in L(H)$. If a subspace $H_{0} \subset H$ reduces $T$, then

$$
\begin{gathered}
P_{\operatorname{ker} T}\left(H_{0}\right)=P_{H_{0}}(\operatorname{ker} T)=\operatorname{ker} T \cap H_{0}, \\
P_{\operatorname{ker} T}\left(H_{0}^{\perp}\right)=P_{H_{0}^{\perp}}(\operatorname{ker} T)=\operatorname{ker} T \cap H_{0}^{\perp} .
\end{gathered}
$$

Proof. Since $H_{0}$ reduces $T$, we have $P_{H_{0}} T=T P_{H_{0}}$ and $P_{H_{0}^{\perp}} T=T P_{H_{0}^{\perp}}$. Thus $P_{H_{0}}(\operatorname{ker} T) \subset \operatorname{ker} T$ and $P_{H_{0}^{\perp}}(\operatorname{ker} T) \subset \operatorname{ker} T$. For each $y \in H_{0}$, since 
$P_{H_{0}^{\perp}} y=0$, it follows that $P_{H_{0}^{\perp}}\left(P_{\operatorname{ker} T} y\right)=-P_{H_{0}^{\perp}}\left(P_{\operatorname{ker} T^{\perp}} y\right)$. Therefore

$$
\left\|P_{H_{0}^{\perp}}\left(P_{\operatorname{ker} T} y\right)\right\|^{2}=\left(P_{H_{0}^{\perp}}\left(P_{\operatorname{ker} T} y\right),-P_{\operatorname{ker} T^{\perp}} y\right)=0 .
$$

Thus $P_{\text {ker } T}\left(H_{0}\right) \subset H_{0}$ and $P_{\operatorname{ker} T}\left(H_{0}^{\perp}\right) \subset H_{0}^{\perp}$.

The subspaces in Theorem 1.2 may be described by the following formulas.

TheOREM 3.3. Let $T \in L(H)$ be a power partial isometry and

$$
H=H_{u} \oplus H_{s} \oplus H_{b} \oplus H_{t}
$$

be the decomposition given in Theorem 1.2 , where $H_{t}=\bigoplus_{k \geq 1} H_{k}$. Then

(i) $H_{b} \oplus H_{t}=\bigoplus_{n \geq 0} T^{* n}(\operatorname{ker} T)$,

(ii) $H_{u} \oplus H_{b}=\bigcap_{n \geq 0} T^{n} H$,

(iii) $H_{s} \oplus H_{t}=\bigoplus_{n \geq 0} T^{n}\left(\operatorname{ker} T^{*}\right)$,

(iv) $H_{u} \oplus H_{s}=\bigcap_{n \geq 0} T^{* n} H$.

Proof. By Theorem 3.1, the operator $T^{*}$ is a power partial isometry. Note that (iii) for $T$ is equivalent to (i) for $T^{*}$. The same relation holds between (iv) and (ii). Therefore it is enough to show (i) and (ii).

For (i) note that since $\left.T\right|_{H_{u} \oplus H_{s}}$ is an isometry, $H_{u} \oplus H_{s}$ is orthogonal to $\operatorname{ker} T$. Therefore, by Lemma 3.2, for $H_{0}=H_{b}$ and $H_{0}^{\perp}=H_{t}$, we obtain

(1) $\quad \operatorname{ker} T=P_{H_{b}}(\operatorname{ker} T) \oplus P_{H_{t}}(\operatorname{ker} T)=\left(\operatorname{ker} T \cap H_{b}\right) \oplus\left(\operatorname{ker} T \cap H_{t}\right)$.

By the geometric structure of the unilateral shift $\left.T^{*}\right|_{H_{b}}$ we have

$$
H_{b}=\bigoplus_{n \geq 0} T^{* n}\left(\operatorname{ker} T \cap H_{b}\right)
$$

Since $\left.T^{*}\right|_{H_{k}}$ is a truncated shift of index $k$,

$$
H_{k}=\bigoplus_{n=0}^{k-1} T^{* n}\left(\operatorname{ker} T \cap H_{k}\right)=\bigoplus_{n \geq 0} T^{* n}\left(\operatorname{ker} T \cap H_{k}\right)
$$

for $k \geq 1$. By Lemma 3.2, for $H_{0}=H_{k}$ and $H_{0}^{\perp}=H_{t} \ominus H_{k}$, we obtain $P_{H_{k}}\left(\operatorname{ker} T \cap H_{t}\right)=\operatorname{ker} T \cap H_{k}$ for all $k \geq 1$, which implies

$$
\operatorname{ker} T \cap H_{t}=\bigoplus_{k \geq 1}\left(\operatorname{ker} T \cap H_{k}\right) \text {. }
$$

Now from (3) and (4) it follows that

$$
\begin{aligned}
H_{t}=\bigoplus_{k \geq 1} H_{k} & \stackrel{(3)}{=} \bigoplus_{k \geq 1} \bigoplus_{n \geq 0} T^{* n}\left(\operatorname{ker} T \cap H_{k}\right) \\
& =\bigoplus_{n \geq 0} T^{* n}\left(\bigoplus_{k \geq 1}\left(\operatorname{ker} T \cap H_{k}\right)\right) \stackrel{(4)}{=} \bigoplus_{n \geq 0} T^{* n}\left(\operatorname{ker} T \cap H_{t}\right)
\end{aligned}
$$


and finally

$$
\begin{aligned}
H_{b} \oplus H_{t} & =\bigoplus_{n \geq 0} T^{* n}\left(\operatorname{ker} T \cap H_{b}\right) \oplus \bigoplus_{n \geq 0} T^{* n}\left(\operatorname{ker} T \cap H_{t}\right) \\
& =\bigoplus_{n \geq 0} T^{* n}\left(\left(\operatorname{ker} T \cap H_{b}\right) \oplus\left(\operatorname{ker} T \cap H_{t}\right)\right) \stackrel{(1)}{=} \bigoplus_{n \geq 0} T^{* n}(\operatorname{ker} T) .
\end{aligned}
$$

For (ii) recall that by $\left[5\right.$, Lemmas 4 and 5] the subspace $\bigcap_{n \geq 0} T^{n} T^{* n} H$ reduces $T^{*}$ to an isometry. Thus $\bigcap_{n \geq 0} T^{n} H=\bigcap_{n \geq 0} T^{n} T^{* n} H \subset H_{u} \oplus H_{b}$. Conversely, since $\left.T^{* n}\right|_{H_{u} \oplus H_{b}}$ is an isometry, $H_{u} \oplus H_{b}$ is orthogonal to ker $T^{* n}$ for any $n \geq 1$. Thus $H_{u} \oplus H_{b} \subset T^{n} H$ for any $n \geq 0$.

The theorem implies the following formulas:

$$
\begin{aligned}
H_{u} & =\bigcap_{n \geq 0} T^{n} H \cap \bigcap_{n \geq 0} T^{* n} H, \\
H_{s} & =\bigcap_{n \geq 0} T^{* n} H \cap \bigoplus_{n \geq 0} T^{n}\left(\operatorname{ker} T^{*}\right), \\
H_{b} & =\bigcap_{n \geq 0} T^{n} H \cap \bigoplus_{n \geq 0} T^{* n}(\operatorname{ker} T), \\
H_{t} & =\bigoplus_{n \geq 0} T^{n}\left(\operatorname{ker} T^{*}\right) \cap \bigoplus_{n \geq 0} T^{* n}(\operatorname{ker} T) .
\end{aligned}
$$

4. Decomposition of pairs of power partial isometries. The class of power partial isometries is not closed under multiplication.

ExAmPle 4.1. Let $\left\{e_{k}, f_{k+1}, g_{k+1}\right\}_{k=0}^{\infty}$ be a set of orthonormal vectors in some complex Hilbert space $\mathbb{H}$. Define a new Hilbert space $H=\bigoplus_{k=0}^{\infty}\left(\mathbb{C} e_{k} \oplus\right.$ $\mathbb{C} f_{k+1} \oplus \mathbb{C} g_{k+1}$ ), and operators (see Fig. 1):

$$
\begin{gathered}
T_{1}\left(e_{k}\right)=T_{2}\left(e_{k}\right)=e_{k+1} \quad \text { for } k \geq 1, \\
T_{1}\left(e_{0}\right)=(\sqrt{2} / 2) e_{1}+(\sqrt{2} / 2) f_{1}, \\
T_{2}\left(e_{0}\right)=(\sqrt{2} / 2) e_{1}+(\sqrt{2} / 2) g_{1}, \\
T_{1}\left(f_{k}\right)=f_{k+1}, \quad T_{2}\left(f_{k}\right)=0 \quad \text { for } k \geq 1, \\
T_{1}\left(g_{k}\right)=0, \quad T_{2}\left(g_{k}\right)=g_{k+1} \quad \text { for } k \geq 1 .
\end{gathered}
$$

One can check that $T_{1}, T_{2}$ commute and are power partial isometries. Since

$$
\operatorname{ker} T_{2}=\bigoplus_{k=1}^{\infty} \mathbb{C} f_{k}, \quad \operatorname{ker} T_{1}=\bigoplus_{k=1}^{\infty} \mathbb{C} g_{k},
$$

and $T_{1} T_{2}\left(\sum_{i=0}^{\infty} \alpha_{i} e_{i}\right)=(\sqrt{2} / 2) \alpha_{0} e_{2}+\sum_{i=1}^{\infty} \alpha_{i} e_{i+2}$ for all $\left\{\alpha_{i}\right\}_{i \geq 0} \subset \mathbb{C}$, we have $\operatorname{ker} T_{1} T_{2}=\operatorname{ker} T_{1} \oplus \operatorname{ker} T_{2}$. Thus $e_{0} \perp \operatorname{ker} T_{1} T_{2}$. Since $\left\|T_{1} T_{2} e_{0}\right\|=$ $\sqrt{2} / 2 \neq\left\|e_{0}\right\|$ the product $T_{1} T_{2}$ is not a partial isometry. 


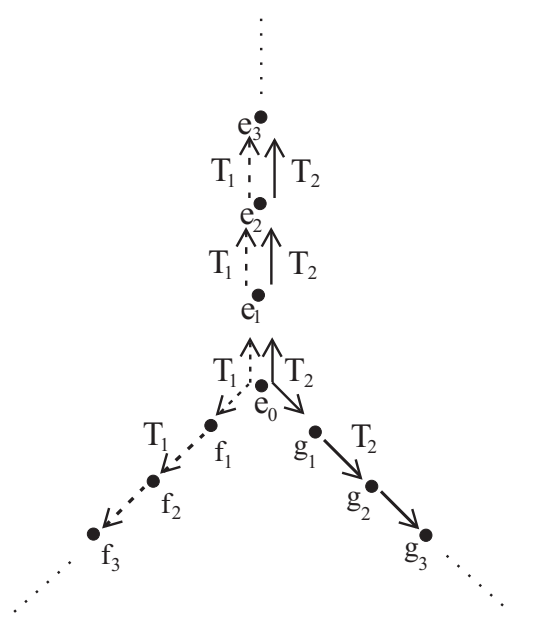

Fig. 1

Recall the result of Halmos and Wallen [5].

LEMma 4.2. If $T_{1}$ and $T_{2}$ are partial isometries, then a necessary and sufficient condition for $T_{1} T_{2}$ to be a partial isometry is that $T_{1}^{*} T_{1}$ and $T_{2} T_{2}^{*}$ commute.

It follows that the product of doubly commuting power partial isometries is a power partial isometry. When power partial isometries only commute, the following holds:

TheOREM 4.3. Let $T_{1}, T_{2} \in L(H)$ be commuting power partial isometries. Then

$$
\begin{aligned}
H_{p}=\overline{\operatorname{Span}}\left\{H_{0} \subset \bigcap_{n \geq 1} \operatorname{ker}\left(T_{2}^{n *} T_{2}^{n} T_{1}^{n} T_{1}^{* n}-T_{1}^{n} T_{1}^{* n} T_{2}^{* n} T_{2}^{n}\right):\right. \\
\left.P_{H_{0}} T_{i}=T_{i} P_{H_{0}} \text { for } i=1,2\right\}
\end{aligned}
$$

is the maximal subspace reducing $T_{1}, T_{2}$ to a pair such that $\left.T_{1} T_{2}\right|_{H_{p}}$ is a power partial isometry.

Proof. By Lemma 4.2 the subspace $H_{p}$ reduces $T_{1}, T_{2}$ to a desired pair, being a closed linear span of such subspaces. The maximality is obvious.

Recall that a co-isometry is an operator whose adjoint operator is an isometry. Note the following property.

Lemma 4.4. Let $L \subset H$ be a subspace reducing commuting power partial isometries $T_{1}, T_{2} \in L(H)$. If at least one of $\left.T_{1}\right|_{L},\left.T_{2}\right|_{L}$ is an isometry or a co-isometry, then $\left.T_{1} T_{2}\right|_{L}$ is a power partial isometry.

Proof. Assume $\left.T_{1}\right|_{L}$ is an isometry. Since $L$ reduces $T_{2}$, we have $\left\|T_{1}^{n} T_{2}^{n}\right\|$ $=\left\|T_{2}^{n} x\right\|$ for any positive integer $n$ and any $x \in L$. On the other hand, $\left\|T_{2}^{n} x\right\|=\|x\|$ for any $x \in\left(\operatorname{ker} T_{1}^{n} T_{2}^{n}\right)^{\perp}$. Thus $\left.T_{1}^{n} T_{2}^{n}\right|_{\left(\operatorname{ker} T_{1}^{n} T_{2}^{n}\right)^{\perp}}$ is an isometry 
for $n \geq 0$. By Theorem 3.1 the case of co-isometry can be deduced from the case of isometry for the adjoint operator.

It follows that any subspace satisfying the conditions of Lemma 4.4 is a subspace of the space $H_{p}$ of Theorem 4.3.

Proposition 4.5. Let $T_{1}, T_{2} \in L(H)$ be commuting power partial isometries. Let $H_{p}$ be the subspace defined in Theorem 4.3. The maximal subspace which reduces $T_{1}, T_{2}$ to a pair of isometries is

$$
H_{\mathrm{Iz}^{2}}=\bigcap_{n \geq 0} T_{1}^{* n} T_{2}^{* n} H_{p}
$$

The maximal subspace which reduces $T_{1}, T_{2}$ to a pair of co-isometries is

$$
H_{\mathrm{CoIz}^{2}}=\bigcap_{n \geq 0} T_{1}^{n} T_{2}^{n} H_{p}
$$

Proof. We will show that $H_{\mathrm{CoIz}^{2}}$ is $T_{1}^{*}$-invariant. The remaining parts of the proof that $H_{\mathrm{CoIz}^{2}}$ and $H_{\mathrm{Iz}^{2}}$ reduce $T_{1}, T_{2}$ are either similar or trivial. Let $y \in H_{\mathrm{CoIz}^{2}}$ and let $n \geq 0$ be an integer. By Lemma 3.2, $P_{\operatorname{ker} T_{1}^{n} T_{2}^{n}}\left(H_{p}\right) \subset H_{p}$ and $P_{\left(\operatorname{ker} T_{1}^{n} T_{2}^{n}\right)^{\perp}}\left(H_{p}\right)=\left(I-P_{\operatorname{ker} T_{1}^{n} T_{2}^{n}}\right)\left(H_{p}\right) \subset H_{p}$. Thus, there is a vector $x \in H_{p} \cap\left(\operatorname{ker} T_{1}^{n} T_{2}^{n}\right)^{\perp}$ such that $y=T_{1}^{n} T_{2}^{n} x$. Hence $\|y\|=\left\|T_{1}^{n} T_{2}^{n} x\right\|=\|x\|$. Thus,

$$
\begin{aligned}
\|x\| \geq\left\|T_{1}^{n-1} T_{2}^{n} x\right\| & \geq\left\|T_{1}^{n} T_{2}^{n} x\right\| \geq\left\|T_{1}^{*} T_{1} T_{1}^{n-1} T_{2}^{n} x\right\| \\
& \geq\left\|T_{1} T_{1}^{*} T_{1} T_{1}^{n-1} T_{2}^{n} x\right\| \stackrel{3.1(6)}{=}\left\|T_{1}^{n} T_{2}^{n} x\right\|=\|y\|=\|x\| .
\end{aligned}
$$

By Theorem 3.1(3), from $\left\|T_{1}^{*} T_{1}\left(T_{1}^{n-1} T_{2}^{n} x\right)\right\|=\left\|T_{1}^{n-1} T_{2}^{n} x\right\|$, we infer that $T_{1}^{*} T_{1}\left(T_{1}^{n-1} T_{2}^{n} x\right)=T_{1}^{n-1} T_{2}^{n} x$.

It follows that

$$
T_{1}^{*} y=T_{1}^{*} T_{1}^{n} T_{2}^{n} x=T_{1}^{n-1} T_{2}^{n} x=T_{1}^{n-1} T_{2}^{n-1}\left(T_{2} x\right) \in T_{1}^{n-1} T_{2}^{n-1} H_{p} .
$$

We obtain the inclusions $T_{1}^{*}\left(T_{1}^{n} T_{2}^{n} H_{p}\right) \subset T_{1}^{n-1} T_{2}^{n-1} H_{p}$ for $n \geq 1$, while for $n=0$ we have $T_{1}^{*} H_{p} \subset H_{p}$. Finally,

$$
T_{1}^{*}\left(\bigcap_{n \geq 0} T_{1}^{n} T_{2}^{n} H_{p}\right) \subset \bigcap_{n \geq 0} T_{1}^{n} T_{2}^{n} H_{p} .
$$

Now, by Theorem 3.3(iii) and the inclusion

$$
\bigcap_{n \geq 0} T_{1}^{* n} T_{2}^{* n} H_{p} \subset \bigcap_{n \geq 0} T_{1}^{* n} H \cap \bigcap_{n \geq 0} T_{2}^{* n} H
$$

the operators $\left.T_{1}\right|_{\bigcap_{n \geq 0} T_{1}^{* n} T_{2}^{* n} H_{p}},\left.T_{2}\right|_{\bigcap_{n \geq 0} T_{1}^{* n} T_{2}^{* n} H_{p}}$ are isometries. To show the maximality, assume that a subspace $L \subset H$ reduces $T_{1}, T_{2}$ to isometries. Then $\left.T_{1}^{n} T_{2}^{n}\right|_{L}$ is an isometry for any positive integer $n$. It follows that $L \subset H_{p}$ 
and $L \perp \operatorname{ker} T_{1}^{n} T_{2}^{n}$ for all $n$. Thus

$$
L \subset \bigcap_{n \geq 0} T_{1}^{* n} T_{2}^{* n} H_{p}
$$

Similarly one can show that $\bigcap_{n \geq 0} T_{1}^{n} T_{2}^{n} H_{p}$ is a maximal subspace reducing $T_{1}, T_{2}$ to co-isometries.

We needed to know that the product $\left.T_{1} T_{2}\right|_{H_{p}}$ is a power partial isometry to prove that $\bigcap_{n>0} T_{1}^{* n} T_{2}^{* n} H_{p}$ reduces the power partial isometries $T_{1}, T_{2}$. In Example 4.1, where the product is not a power partial isometry, a similar subspace $\bigcap_{n \geq 0} T_{1}^{* n} T_{2}^{* n} H$ does not reduce power partial isometries.

THEOREM 4.6. Let $T_{1}, T_{2} \in L(H)$ be commuting power partial isometries, and let $H_{p}$ be the maximal subspace reducing $T_{1}, T_{2}$ such that $\left.T_{1} T_{2}\right|_{H_{p}}$ is a power partial isometry. Then the maximal subspace reducing $T_{1}, T_{2}$ to a pair of unitary operators is

$$
H_{u u}=\bigcap_{n \geq 0} T_{1}^{n} T_{2}^{n} H_{p} \cap \bigcap_{n \geq 0} T_{1}^{* n} T_{2}^{* n} H_{p} .
$$

Proof. By Proposition 4.5, $H_{u u}$ reduces $T_{1}, T_{2}$, and the restrictions $\left.T_{1}\right|_{H_{u u}}$, $\left.T_{2}\right|_{H_{u u}},\left.T_{1}^{*}\right|_{H_{u u}},\left.T_{2}^{*}\right|_{H_{u u}}$ are isometries. Therefore $\left.T_{1}\right|_{H_{u u}},\left.T_{2}\right|_{H_{u u}}$ are unitary. The maximality of $H_{u u}$ is obvious by Proposition 4.5, because a unitary operator is an isometry and a co-isometry.

Recall [6] that a pair of commuting contractions $T_{1}, T_{2} \in L(H)$ is said to belong to $K_{0}$. when $\lim _{n \rightarrow \infty}\left\|T_{1}^{n} T_{2}^{n} x\right\|=0$ for every $x \in H$. A pair $T_{1}, T_{2}$ is said to belong to $K_{.0}$ if the pair $T_{1}^{*}, T_{2}^{*}$ belongs to $K_{0}$.. The intersection of $K_{0}$. and $K_{.0}$ is called $K_{00}$.

Proposition 4.7. Let $T_{1}, T_{2} \in L(H)$ be commuting power partial isometries and $H_{p}$ be the subspace defined in Theorem 4.3. Then

$$
H_{p t}=H_{p} \ominus \overline{\operatorname{Span}}\left\{\bigcap_{n \geq 0} T_{1}^{* n} T_{2}^{* n} H_{p}, \bigcap_{n \geq 0} T_{1}^{n} T_{2}^{n} H_{p}\right\}
$$

is a maximal subspace reducing $T_{1}, T_{2}$ such that $\left.T_{1} T_{2}\right|_{H_{p t}}$ is an orthogonal sum of truncated shifts of some indices.

Proof. From Theorem 4.3, we infer that $\left.T_{1} T_{2}\right|_{H_{p}}$ is a power partial isometry. By Theorem 3.1(3), $\left\{\left.T_{1}^{* n} T_{2}^{* n} T_{1}^{n} T_{2}^{n}\right|_{H_{p}}\right\}_{n \in \mathbb{Z}_{+}}$is a decreasing sequence of orthogonal projections onto $\mathcal{R}\left(\left.T_{1}^{* n} T_{2}^{* n}\right|_{H_{p}}\right)$. Therefore, $\left\{\left.T_{1}^{* n} T_{2}^{* n} T_{1}^{n} T_{2}^{n}\right|_{H_{p}}\right\}_{n \in \mathbb{Z}_{+}}$ converges in the strong operator topology in $L\left(H_{p}\right)$ to the orthogonal projection onto $\bigcap_{n \geq 0} T_{1}^{* n} T_{2}^{* n} H_{p}$. By Proposition 4.5, the latter space is a maximal subspace of $\bar{H}_{p}$ reducing $T_{1}, T_{2}$ to isometries. By definition, $H_{p t}$ is orthogonal to $\bigcap_{n \geq 0} T_{1}^{* n} T_{2}^{* n} H_{p}$ and is a subspace of $H_{p}$. Thus $T_{1}^{* n} T_{2}^{* n} T_{1}^{n} T_{2}^{n} x$ converges 
to zero for any $x \in H_{p t}$. Since $T_{1}, T_{2}$ are contractions, we have

$$
\begin{aligned}
\lim _{n \rightarrow \infty}\left\|T_{1}^{n} T_{2}^{n} x\right\| & \stackrel{3.1(6)}{=} \lim _{n \rightarrow \infty}\left\|T_{1}^{n} T_{2}^{n} T_{1}^{* n} T_{2}^{* n} T_{1}^{n} T_{2}^{n} x\right\| \\
& \leq \lim _{n \rightarrow \infty}\left\|T_{1}^{* n} T_{2}^{* n} T_{1}^{n} T_{2}^{n} x\right\|=\left\|\lim _{n \rightarrow \infty} T_{1}^{* n} T_{2}^{* n} T_{1}^{n} T_{2}^{n} x\right\|=0 .
\end{aligned}
$$

In the same way one can show that

$$
\lim _{n \rightarrow \infty}\left\|T_{1}^{* n} T_{2}^{* n} x\right\|=0 .
$$

Thus the pair $\left.T_{1}\right|_{H_{p t}},\left.T_{2}\right|_{H_{p t}}$ belongs to the class $K_{00}$. Theorem 1.2 can be applied to the product $\left.T_{1} T_{2}\right|_{H_{p t}}$. Note that since $\left.T_{1}\right|_{H_{p t}},\left.T_{2}\right|_{H_{p t}}$ is in $K_{00}$, there is no nontrivial subspace reducing $\left.T_{1} T_{2}\right|_{H_{p t}}$ to an isometry or a coisometry. Therefore, $\left.T_{1} T_{2}\right|_{H_{p t}}$ is an orthogonal sum of truncated shifts of some indices. To show the maximality, consider a subspace $L \subset H$ reducing $T_{1}, T_{2}$ to a pair such that $\left.T_{1} T_{2}\right|_{L}$ is an orthogonal sum of truncated shifts of some indices. Then $\left.T_{1} T_{2}\right|_{L}$ is a power partial isometry and $L \subset H_{p}$. Since $\left.T_{1} T_{2}\right|_{L}$ is a sum of truncated shifts, $L$ is orthogonal to the subspaces $\bigcap_{n \geq 0} T_{1}^{* n} T_{2}^{* n} H_{p}$ and $\bigcap_{n \geq 0} T_{1}^{n} T_{2}^{n} H_{p}$ which reduce $T_{1} T_{2}$ to an isometry and a co-isometry, respectively. Thus $L \subset H_{p t}$, which finishes the proof.

We can now state the main decomposition theorem.

TheOREM 4.8. Let $T_{1}, T_{2} \in L(H)$ be commuting power partial isometries. There is a unique decomposition

$$
\begin{aligned}
H= & H_{u u} \oplus H_{u s} \oplus H_{u b} \oplus \bigoplus_{k \geq 1} H_{u k} \\
& \oplus H_{s u} \oplus H_{b u} \oplus \bigoplus_{k \geq 1} H_{k u} \oplus H_{\mathrm{iz}^{2}} \oplus H_{\mathrm{coiz}^{2}} \oplus H_{K_{00}} \oplus H_{\neg p},
\end{aligned}
$$

where $H_{u u}, H_{u s}, H_{u b}, H_{s u}, H_{b u}, H_{\mathrm{iz}^{2}}, H_{\mathrm{coiz}^{2}}, H_{K_{00}}, H_{\neg p}$ and $H_{u k}, H_{k u}$ for $k \geq 1$ are maximal subspaces reducing, respectively:

- $H_{u u}-T_{1}, T_{2}$ to unitary operators,

- $H_{u s}-T_{1}$ to a unitary operator, $T_{2}$ to a unilateral shift,

- $H_{u b}-T_{1}$ to a unitary operator, $T_{2}$ to a backward shift,

- $H_{u k}-T_{1}$ to a unitary operator, $T_{2}$ to a truncated shift of index $k$,

- $H_{s u}-T_{1}$ to a unilateral shift, $T_{2}$ to a unitary operator,

- $H_{b u}-T_{1}$ to a backward shift, $T_{2}$ to a unitary operator,

- $H_{k u}-T_{1}$ to a truncated shift of index $k, T_{2}$ to a unitary operator,

- $H_{\mathrm{iz}^{2}}-T_{1}, T_{2}$ to a strongly completely nonunitary pair of isometries which is a weak bi-shift,

- $H_{\mathrm{coiz}^{2}}-T_{1}, T_{2}$ to a strongly completely nonunitary pair of co-isometries,

- $H_{K_{00}}-T_{1}, T_{2}$ to a strongly completely nonunitary pair of contractions such that $T_{1} T_{2}$ is an orthogonal sum of truncated shifts of some indices, 
- $H_{\neg p}$ is a maximal subspace not containing a proper subspace reducing $T_{1}, T_{2}$ to a pair such that the product is a power partial isometry.

Proof. By Theorem 2.1, we obtain the decomposition $H=H_{u u} \oplus H_{u \neg u} \oplus$ $H_{\neg u u} \oplus H_{\neg(u u)}$. It can be shown as in Theorem 2.1 that if one of the commuting operators is unitary, they doubly commute. Therefore, each of the pairs $\left.T_{1}\right|_{H_{\neg u u}},\left.T_{2}\right|_{H_{\neg u u}}$ and $\left.T_{1}\right|_{H_{u \neg u}},\left.T_{2}\right|_{H_{u \neg u}}$ doubly commutes and Theorem 1.4 can be applied. This way we obtain a more detailed result: $H=H_{u u} \oplus H_{u s} \oplus H_{u b} \oplus \bigoplus_{k \geq 1} H_{u k} \oplus H_{s u} \oplus H_{b u} \oplus \bigoplus_{k \geq 1} H_{k u} \oplus H_{\neg(u u)}$. Following Theorem 4.3, denote by $H_{p}$ a maximal subspace reducing $T_{1}, T_{2}$ such that $\left.T_{1} T_{2}\right|_{H_{p}}$ is a power partial isometry. Now, set $H_{\neg p}=H \ominus H_{p}$, which, by Lemma 4.4, is a subspace of $H_{\neg(u u)}$. By Proposition 4.5, there are maximal subspaces reducing $T_{1}, T_{2}$ to a pair of isometries (denoted $H_{\mathrm{Iz}^{2}}$ ) and a pair of co-isometries (denoted $H_{\mathrm{CoIz}^{2}}$ ). Now set

$$
\begin{aligned}
H_{\mathrm{iz}^{2}} & =P_{H_{\neg(u u)}} H_{\mathrm{Iz}^{2}}=H_{I z^{2}} \ominus\left(H_{u u} \oplus H_{u s} \oplus H_{s u}\right), \\
H_{\mathrm{coiz}^{2}} & =P_{H_{\neg(u u)}} H_{\mathrm{CoIz}^{2}}=H_{\mathrm{CoIz}^{2}} \ominus\left(H_{u u} \oplus H_{u b} \oplus H_{b u}\right), \\
H_{K_{00}} & =H_{\neg(u u)} \ominus\left(H_{\neg p} \oplus H_{i z^{2}} \oplus H_{\mathrm{coiz}^{2}}\right) \\
& =H_{\neg(u u)} \cap\left(H_{p} \ominus \overline{\operatorname{Span}}\left\{H_{I z^{2}}, H_{\mathrm{CoIz}^{2}}\right\}\right) .
\end{aligned}
$$

By Propositions 4.5, 4.7 and Theorem 2.1, $H_{K_{00}}$ is a maximal subspace reducing $T_{1}, T_{2}$ to a strongly completely nonunitary pair such that $\left.T_{1} T_{2}\right|_{H_{K_{00}}}$ is an orthogonal sum of truncated shifts of some indices. The subspace $H_{K_{00}}$ has been obtained as the orthogonal complement of all previous subspaces. The decomposition is complete.

The decomposition in Theorem 4.8 can easily be made more detailed to yield, in the case of doubly commuting power partial isometries, the same decomposition as in Theorem 1.4. Instead of doing this, which would unreasonably increase the number of decomposing subspaces, we compare these two decompositions.

REMARK 4.9. Let $T_{1}, T_{2} \in L(H)$ be power partial isometries. Consider the decomposition defined in Theorem 4.8, using the same notation. Denote by $H^{d c}$ a maximal subspace reducing $T_{1}, T_{2}$ to a doubly commuting pair. Such a subspace can be found as in Theorem 2.1. The pair $\left.T_{1}\right|_{H^{d c}},\left.T_{2}\right|_{H^{d c}}$ can be decomposed using Theorem 1.4. We use the same notation for subspaces as in Theorem 1.4 with an additional upper index ${ }^{d c}$ (e.g. $\left.H_{u s}^{d c}\right)$. Recall that if one of the operators $T_{1}, T_{2}$ is unitary, they doubly commute. It follows that $H_{u \alpha}^{d c}=H_{u \alpha}, H_{\alpha u}^{d c}=H_{\alpha u}$ for $\alpha=u, s, b, 1,2, \ldots$ The subspaces $H_{b s}^{d c}, H_{s b}^{d c}, H_{k s}^{d c}, H_{k b}^{d c}, H_{b l}^{d c}, H_{s l}^{d c}, H_{k l}^{d c}$ are contained in $H_{K_{00}}$ for any $k, l \in \mathbb{Z}_{+}$. To the operators $\left.T_{1}\right|_{H_{\mathrm{iz}} 2},\left.T_{2}\right|_{H_{\mathrm{iz}}{ }^{2}}$ and adjoint operators $\left.T_{1}^{*}\right|_{\mathrm{coiz}^{2}},\left.T_{2}^{*}\right|_{H_{\text {coiz }}{ }^{2}}$, we can apply the decomposition for a pair of commuting isometries 
([8, Theorem 3.10] or more detailed [1, Theorem 3.13]). We obtain $H_{s s}^{d c}=H_{s s}$ and $H_{b b}^{d c}=H_{b b}$, where $H_{s s}$ is a subspace of $H_{\mathrm{iz}^{2}}$ reducing $T_{1}, T_{2}$ to doubly commuting unilateral shifts. Similarly, $H_{b b}$ is a subspace of $H_{\text {coiz }^{2}}$ reducing $T_{1}^{*}, T_{2}^{*}$ to doubly commuting unilateral shifts.

An example of an $H_{K_{00}}$-type pair of power partial isometries can be easily obtained by taking a truncated shift of some index and any other suitable operator. More interesting is the following example, where none of the operators has a truncated shift part, but their product is a sum of truncated shifts of some indices.

Example 4.10. Let $H=\bigoplus_{(i, j) \in J} \mathbb{C} e_{i, j}$ be a Hilbert space where $J=$ $\left\{(i, j) \in \mathbb{Z}^{2}:(i \geq 0\right.$ or $j \geq 0)$ and $(i \leq 5$ or $\left.j \leq 5)\right\}$ and $\left\{e_{(i, j)}\right\}_{(i, j) \in J}$ are orthonormal vectors. Define operators $T_{1}, T_{2} \in L(H)$ as follows:

$$
\begin{array}{ll}
T_{1}\left(e_{5, j}\right)=0 & \text { for } j>5, \\
T_{1}\left(e_{i, j}\right)=e_{i+1, j} & \text { for the remaining }(i, j) \in J, \\
T_{2}\left(e_{i, 5}\right)=0 & \text { for } i>5, \\
T_{2}\left(e_{i, j}\right)=e_{i, j+1} & \text { for the remaining }(i, j) \in J .
\end{array}
$$

One can check that the operators commute and both are orthogonal sums of a unilateral shift, a backward shift and a bilateral shift.

Thus, they are power partial isometries. We have $T_{1} T_{2}\left(e_{5, j}\right)=0$ for every $j \geq 5, T_{1} T_{2}\left(e_{i, 5}\right)=0$ for every $i \geq 5$, and $T_{1} T_{2}\left(e_{i, j}\right)=e_{i+1, j+1}$ if $i \leq 4$ or $j \leq 4$. Fix $k \in \mathbb{Z}_{-} \cup\{0\}$. Then

$$
E_{k}=\left\langle e_{k, 0} ; e_{k+1,1} ; \ldots ; e_{5,5-k} ; e_{0, k} ; e_{1, k+1} ; \ldots ; e_{5-k, 5}\right\rangle
$$

is a maximal subspace reducing $T_{1} T_{2}$ to a truncated shift of index $5-k$. 
Since $H=\bigoplus_{k \leq 0} E_{k}$, the product $T_{1} T_{2}$ is an orthogonal sum of truncated shifts with all indices at least 5 .

\section{References}

[1] Z. Burdak, On decomposition of pairs of commuting isometries, Ann. Polon. Math. 84 (2004), 121-135.

[2] X. Catepillán, M. Ptak and W. Szymański, Multiple canonical decompositions of families of operators and a model of quasinormal families, Proc. Amer. Math. Soc. 121 (1994), 1165-1172.

[3] X. Catepillán and W. Szymański, A model of a family of power partial isometries, Far East J. Math. Sci. 4 (1996), 117-124.

[4] J. B. Conway, Subnormal Operators, Pitman, London, 1981.

[5] P. R. Halmos and L. J. Wallen, Powers of partial isometries, J. Math. Mech. 19 (1970), 657-663.

[6] M. Kosiek and A. Octavio, Wold-type extension for $N$-tuples of commuting contractions, Studia Math. 137 (1999), 81-91.

[7] H. Langer, Ein Zerspaltungssatz für Operatoren im Hibertraum, Acta Math. Acad. Sci. Hungar. 12 (1961), 441-445.

[8] D. Popovici, A Wold-type decomposition for commuting isometric pairs, Proc. Amer. Math. Soc. 132 (2004), 2303-2314,

[9] M. Słociński, Models for doubly commuting contractions, Ann. Polon. Math. 45 (1985), 23-42.

[10] I. Suciu, On the semi-groups of isometries, Studia Math. 30 (1968), 101-110.

[11] B. Sz.-Nagy et C. Foiaş, Sur les contractions de l'espace de Hilbert, IV, Acta Sci. Math. (Szeged) 21 (1960), 251-259.

[12] H. Wold, A Study in the Analysis of Stationary Time Series, Almkvist and Wiksell, Stockholm, 1954.

Institute of Mathematics

University of Agriculture

Mickiewicza 24/28

30-059 Kraków, Poland

E-mail: rmburdak@cyf-kr.edu.pl

Received March 15, 2006

Revised version April 24, 2007

(5884) 